\title{
Article \\ Association of SARS-CoV-2 Vaccinations with SARS-CoV-2 Infections, ICU Admissions and Deaths in Greece
}

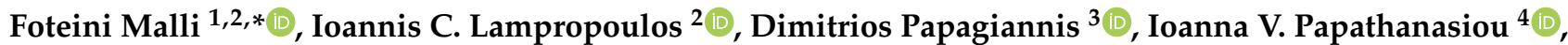 \\ Zoe Daniil $^{2}$ and Konstantinos I. Gourgoulianis ${ }^{2}$ (D)
}

1 Respiratory Disorders Laboratory, Faculty of Nursing, University of Thessaly, 41500 Larissa, Greece

2 Respiratory Medicine Department, Faculty of Medicine, University of Thessaly, 41500 Larissa, Greece; i.ch.lampropoulos@gmail.com (I.C.L.); zdaniil@med.uth.gr (Z.D.); kgourg@med.uth.gr (K.I.G.)

3 Public Health \& Vaccines Laboratory, School of Health Sciences, Faculty of Nursing, University of Thessaly, 41110 Larissa, Greece; dpapajohn@uth.gr

4 Community Nursing Laboratory, Faculty of Nursing, University of Thessaly, 41500 Larissa, Greece; iopapathanasiou@uth.gr

* Correspondence: mallifoteini@yahoo.gr; Tel.: +30-2413502896

check for

updates

Citation: Malli, F.; Lampropoulos,

I.C.; Papagiannis, D.; Papathanasiou,

I.V.; Daniil, Z.; Gourgoulianis, K.I.

Association of SARS-CoV-2

Vaccinations with SARS-CoV-2

Infections, ICU Admissions and

Deaths in Greece. Vaccines 2022, 10,

337. https://doi.org/10.3390/

vaccines10020337

Academic Editor: Yutaka Ueda

Received: 5 January 2022

Accepted: 15 February 2022

Published: 21 February 2022

Publisher's Note: MDPI stays neutral with regard to jurisdictional claims in published maps and institutional affiliations.

Copyright: () 2022 by the authors Licensee MDPI, Basel, Switzerland. This article is an open access article distributed under the terms and conditions of the Creative Commons Attribution (CC BY) license (https:// creativecommons.org/licenses/by/ $4.0 /)$.

\begin{abstract}
The available coronavirus disease 2019 (COVID-19) vaccines have shown their effectiveness in clinical trials. We aimed to assess the real-world effects of SARS-CoV-2 vaccinations in Greece. We combined national data on vaccinations, SARS-CoV-2 cases, COVID-19-related ICU admissions and COVID-19-related deaths. We observed 3,367,673 vaccinations (30.68\% of the Greek population), 278,821 SARS-CoV-2 infections and 7401 COVID-19-related deaths. The vaccination rate significantly increased from week 2 to week 6 by $85.70 \%$, and from week 7 to 25 by $15.65 \%$. The weekly mean of SARS-CoV-2 cases, COVID-19 ICU patients and COVID-19 deaths markedly declined as vaccination coverage accumulated. The rate of SARS-CoV-2 cases increased significantly from week 2 to week 13 by $16.15 \%$, while from weeks $14-25$ the rate decreased significantly by $13.50 \%$. The rate of COVID-19-related ICU admissions decreased significantly by $7.41 \%$ from week 2 to week 4 , increased significantly by $17.22 \%$ from weeks $5-11$, then decreased significantly from weeks $17-20$, by $11.99 \%$, and from weeks $21-25$, by $16.77 \%$. The rate of COVID-19-related deaths increased significantly from week 2 to week 15 by $12.08 \%$ and decreased significantly by $16.58 \%$ from weeks $16-25$. The data from this nationwide observational study underline the beneficial impact of the national vaccination campaign in Greece, which may offer control of the SARS-CoV-2 pandemic.
\end{abstract}

Keywords: COVID-19; SARS-CoV-2; vaccination

\section{Introduction}

Coronavirus Disease 19 (COVID-19) has emerged as an ongoing pandemic that has resulted in more than 5 million deaths worldwide [1]. The fatality ratio of COVID-19 cases (i.e., the number of deaths divided by the number of diagnoses) ranges by region from $19.48 \%$ to below 1\% [2]. Mortality is increased in elderly patients, males and subjects with comorbidities such as diabetes, arterial hypertension and cardiovascular disease [3].

In response to the pandemic, several attempts have been made to control the dissemination of the SARS-CoV-2 infection in order to reduce COVID-19-related morbidity and mortality. Recently, authorization of COVID-19 vaccines took place, soon after the publication of the initial phase 3 trials [4], and to date more than 8 billion vaccine doses have been administered worldwide [2]. In randomized clinical trials, their rates of effectiveness against symptomatic COVID-19 were 95\% for the BNT162b2 vaccine (Pfizer BioNTech), 70.4\% for the ChAdOx1 nCoV-19 vaccine (AZD1222, Astra Zeneca) and 94.1\% for the mRNA-1273 SARS-CoV-2 vaccine (Moderna) [5-7]. The emergency development of COVID-19 vaccines, as well as beliefs that these vaccines are not effective, have led to negative attitudes and vaccine hesitancy worldwide [8,9]. Large epidemiological studies are warranted in order 
to estimate the real-world effectiveness of COVID-19 vaccines in order to overcome the aforementioned barriers to vaccine acceptance.

The first case of COVID-19 in Greece was observed on the 26 February 2020 [10]. The vaccination campaign in Greece began on the 4 January 2021, during the second pandemic wave [11]. A third pandemic wave occurred in Greece during March 2021 and the total COVID-19 cases exceeded 200,000 [12]. By the end of May 2021, Greece recorded a total of 400,000 COVID-19 cases and entered the resolution phase of the third wave. The temporal impact of the vaccination campaign in the course of the pandemic in Greece has not been reported previously.

In the present study we aim to address the effectiveness estimates for COVID-19 vaccinations in the first 25 weeks of the national vaccination campaign in Greece. To this end, we associated the rate of vaccinations against SARS-CoV-2 with the rate of new SARS-CoV-2 cases, COVID-19-related ICU admissions and COVID-19-related deaths.

\section{Materials and Methods}

\subsection{Study Population}

We used national surveillance data to address the association of SARS-CoV-2 vaccination on COVID-19 outcomes (i.e., new SARS-CoV-2 cases, COVID-19-related ICU admissions and COVID-19-related deaths). We analyzed data from the 1st to the 25th week of the vaccination campaign. Data concerning vaccinations were retrieved from the European Centre for Disease Prevention and Control (ECDC) [13]. Vaccination trend analysis included data from fully completed vaccinations. Data about COVID-19 infections and outcomes were retrieved from the National Public Health Organization (NPHO) of Greece [14].

The nationwide vaccination campaign in Greece began on the 4 January 2021. The vaccination was initially applied to health care workers (1st week), subjects older than 85 years old (2nd week), while on the 3rd week of vaccinations, vaccine availability extended to subjects in the age group 80-84 years. At week 6, vaccination was extended to citizens of 60-64 years old and 75-79 years old. At week 13, vaccine availability was extended to ages 65-69 years, at week 16, to ages 50-59 years and at week 17, to ages $30-49$ years. The vaccination program was started with the Pfizer/BioNTech vaccine and later the Oxford/Astra Zeneca, Moderna and Johnson \& Johnson were subsequentially introduced. Vaccination was free of charge for the subjects vaccinated.

The three outcomes assessing vaccination effectiveness were as follows: 1. SARS$\mathrm{CoV}-2$ cases were defined as laboratory confirmed SARS-CoV-2 cases (symptomatic or asymptomatic); 2. Severe COVID-19 patients were defined as those admitted to the ICU; 3. Deaths attributed to COVID-19 were defined as deaths in patients with confirmed COVID-19.

The National Public Health Organization of Greece (NPHO) collects the data from all the diagnostic laboratories and reports all the daily laboratory confirmed cases of SARS-CoV-2 infections. All hospitals provided daily updates on the number of new cases, ICU admissions, deaths, etc., and the data are provided to the National database.

ECDC data on vaccinations are reported for the following age groups: 18-24 years, $25-49$ years, $50-59$ years, $60-69$ years, $70-79$ years and $\geq 80$ years, while NPHO reports data for the age groups 0-17 years, 18-39 years, $40-64$ years and $\geq 65$ years. In order to perform subgroup analysis of the vaccination effect according to age, we grouped the data for patients aged $<69$ years old in ECDC with those of patients aged $\leq 65$ years in NPHO files while data from patients $\geq 70$ years in ECDC and $\geq 65$ years in NPHO were also grouped together.

Ethics approval was not applicable since we revised publicly available national surveillance data. No identifiable demographic or personal data were used in the present study. 


\subsection{Statistical Analysis}

Data are presented as absolute numbers or as percentages. We used joinpoint regression modelling in order to assess the variation in the trends in vaccination rates, SARS-CoV2 cases, COVID-19-related ICU admissions and COVID-19-related deaths. The joinpoint regression model investigates the combinations of trends that result in a statistically significantly better fit to a data series than a single-trend line fitted using Poisson regression or time-series models [15]. With this procedure, one may determine the number of joinpoints that are sufficient for the estimation of significant alterations in incidence trends over time. The Joinpoint Regression Program (3.5.2) and SPSS 20 were used to analyze the data. A statistically significant joinpoint was set at $p<0.05$.

\section{Results}

The first vaccination efforts in Greece started on the 27 December 2020 with sparse vaccinations, whilst the nationwide vaccination campaign began on the 4 January 2021 (week 1) with the vaccination of healthcare workers, and was extended on the 19 January to persons aged $\geq 85$ years. The vaccination campaign started while Greece was under a nationwide lockdown, which had begun on the 7 November 2020. Phased recession of the restriction measures occurred on the 11 January 2021 (the 2 nd week of the vaccination campaign) with the opening of school facilities.

During the study period, there were 278,821 new SARS-CoV-2 infections and 7401 COVID-19-related deaths. At the end of the study period, 3,367,673 full vaccinations occurred $(19.49 \%$ in aged $<70$ years), which amounts to $30.68 \%$ of the Greek population. Table 1 presents the weekly distribution of vaccinations, new SARS-CoV-2 cases, ICU admissions due to COVID-19, COVID-19-related deaths and the ratio of COVID-19related ICU admissions/SARS-CoV-2 cases and the ratio of COVID-19-related deaths/SARSCoV-2 cases.

The course of vaccinations by age group from week 1 to week 25 is presented in Figure 1. According to the results of the joinpoint analysis, for the age group of 18-24 years, there was a significant increase in the rate of vaccinations by $9.63 \%$ (Cis: $4.7-14.8$ ) from week 2 to week 19, which was followed by a significant increase by $148.21 \%$ (Cis: $46.5-320.5$ ) from week 20 to week 22 . The rate decreased significantly by $31.96 \%$ (Cis: $-51.6--4.3$ ) from week 23 to week 25 (Figure 1). For subjects 25-49 years, we observed a significant decrease in the rate of vaccinations by $9.91 \%$ (Cis: -17.5--1.6) from week 2 to week 13, which was followed by a significant increase by $37.79 \%$ (Cis: $24.8-52.2$ ) from week 14 to week 22 , while during weeks $23-25$ the rate of vaccinations did not significantly differ. For the age group 50-59 years, vaccination displayed a significantly decreasing trend from week 2 to week 12 by $17.29 \%$ (Cis: $-30.5--1.6$ ). The rate significantly increased by $58.12 \%$ from week 13 to 22 (Cis: $43.3-74.4$ ) and significantly decreased by $43.72 \%$ (Cis: $-67.5--2.6$ ) from week 23 to 25 . For the age group 60-69 years, we did not observe statistically significant changes in vaccination rates from week 2 to 13 and from week 19 to 25 , while the rate significantly increased by $164.52 \%$ (Cis: $26.6-452.9$ ) for week 14 to 18 . For the persons aged 70-79 years, we observed a significant increase of $30.08 \%$ (Cis: 9.6-54.4) for the weeks 2 to 17 and a significant decrease of $20.57 \%$ (Cis: $-33.1--5.6$ ) for the weeks 18 to 25 . Finally, for persons aged $\geq 80$ years, there was a significantly decreasing trend in the vaccination rate from week 2 to week 25 by $9.3 \%$ (Cis: $-13.0--5.4$ ). 
Table 1. Weekly distribution of vaccinations, new SARS-CoV-2 cases, ICU admissions due to COVID19, COVID-19-related deaths and the ratio of COVID-19-related ICU admissions/SARS-CoV-2 cases and the ratio of COVID-19-related deaths/SARS-CoV-2 cases. The data on vaccinations are presented cumulatively. The data are presented as absolute numbers.

\begin{tabular}{|c|c|c|c|c|c|c|c|c|c|c|c|c|c|c|c|c|c|c|}
\hline \multirow[t]{2}{*}{ Weel } & \multicolumn{3}{|c|}{ Vaccinations } & \multicolumn{3}{|c|}{$\begin{array}{l}\text { New SARS } \\
\text { CoV-2 Cases } \\
\text { Per Week }\end{array}$} & \multicolumn{3}{|c|}{$\begin{array}{c}\text { ICU } \\
\text { Admissions } \\
\text { Per Week }\end{array}$} & \multicolumn{3}{|c|}{$\begin{array}{c}\text { Deaths Per } \\
\text { Week }\end{array}$} & \multicolumn{3}{|c|}{$\begin{array}{c}\text { COVID-19- } \\
\text { Related ICU } \\
\text { Admissions/SARS- } \\
\text { CoV-2 Cases }\end{array}$} & \multicolumn{3}{|c|}{$\begin{array}{l}\text { COVID-19- } \\
\text { Related } \\
\text { Death/SARS- } \\
\text { CoV-2 Cases }\end{array}$} \\
\hline & $\begin{array}{c}\text { All } \\
\text { Ages }\end{array}$ & $<70$ & $>70$ & $\begin{array}{l}\text { All } \\
\text { Ages }\end{array}$ & 0 & & $\begin{array}{l}\text { All } \\
\text { Ages }\end{array}$ & $<70$ & $>70$ & $\begin{array}{l}\text { All } \\
\text { Ages }\end{array}$ & & $>$ & $\begin{array}{l}\text { All } \\
\text { Ages }\end{array}$ & 70 & $>70$ & $\begin{array}{l}\text { All } \\
\text { Ages }\end{array}$ & $<70$ & $>70$ \\
\hline 2 & 0 & 0 & 0 & & & & & & & 2 & 41 & & & & & & & \\
\hline 3 & & & & & & & & & & 177 & & & & & & & & \\
\hline 4 & 38,928 & & & & & & & 92 & & 150 & 26 & & & & & & & \\
\hline 5 & & & & & & & & & & 176 & 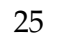 & & & & & & & \\
\hline 6 & & & & & & & & & & 154 & -0 & & & & & & & \\
\hline 7 & & & & & & & & & & 172 & & & & & & & & \\
\hline 8 & & & & 275 & & & & & & 206 & 23 & & & & & & & 00 \\
\hline 9 & & & & & & & & & 284 & 254 & 26 & & 33 & & & & & \\
\hline 10 & & & & & & & & & 355 & 333 & 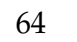 & & & & & 21 & & \\
\hline 11 & & & & & & & & & & 371 & & & & & & & & \\
\hline 12 & & & & & & & & & & 418 & 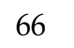 & & & & & 24 & & \\
\hline 13 & & & & & & & & & 477 & 280 & 46 & & 58 & & & 21 & & 105 \\
\hline 14 & & & & & & & & & 482 & 725 & 94 & & 0.028 & & & 0.026 & & 0.135 \\
\hline 15 & & & & & & & & & & 577 & & & & & & & & \\
\hline 16 & & & & & & & & & & 545 & & & & & & & & \\
\hline 17 & & & & 2 & & & 8 & & 496 & 446 & 86 & 360 & 0.061 & & 0.240 & 33 & & 0.174 \\
\hline 18 & 15,201 & & & & & & 728 & 301 & 427 & 576 & 118 & 458 & 0.047 & & 0.197 & 0.037 & & 0.212 \\
\hline 19 & & & & & & & & & 375 & 386 & 74 & 312 & 0.044 & & & 0.026 & & 101 \\
\hline 20 & & & & & & & & & 337 & 357 & 67 & & & & & & & 229 \\
\hline 21 & 43 & 938 & & 1,483 & 10,521 & 96 & 497 & 204 & 293 & 282 & 59 & 223 & 0.043 & 0.019 & 0.305 & 0.025 & 0.006 & 0.232 \\
\hline 22 & & 1,211 & & 063 & & 67 & 398 & 157 & 241 & 223 & 44 & 179 & 0.049 & 0.021 & 0.357 & 0.028 & 0.006 & 0.265 \\
\hline 23 & $2,734,947$ & $1,596,852$ & & 551 & & 49 & 358 & 141 & 217 & 145 & 25 & 120 & 0.064 & 0.028 & 0.441 & 0.026 & 0.005 & 0.244 \\
\hline 24 & & & & & & & & & 166 & 126 & 20 & & & & & & & 0.416 \\
\hline 25 & $3,307,673$ & $2,089,110$ & $1,218,563$ & 2584 & 2427 & 15 & 23 & 95 & 136 & 116 & 27 & 89 & 0.089 & 0.039 & 0.866 & 0.045 & 0.011 & 0.567 \\
\hline
\end{tabular}

Weekly trends in vaccinations in persons aged $\geq 70$ years in comparison with those $<70$ years are presented in Figure 2. For subjects aged $<70$ years, we did not observe significant differences in the vaccination rate from week 2 to week 6 , while for week 7 to week 15 , we observed a significant increase in the vaccination rate by $7.03 \%$ (Cis: $3.8-10.4$ ), which was followed by a significant increase of 33.07\% (Cis: 29.7-36.6) for week 16 to 22 and a significant increase of $13.61 \%$ (Cis: 4.8-23.1) for week 23 to 25 (Figure 2). For subjects $\geq 70$ years, vaccination rates were not statistically significantly different between week 2 and week 5 . The vaccination rate significantly increased by $46.49 \%$ (Cis: $5-104.4$ ) for week 6 to week 8 , by $14.95 \%$ (Cis: 13-16.9) for week 9 to 18 and by 3.92\% (Cis: $1.8-6$ ) for week 19 to 25. 


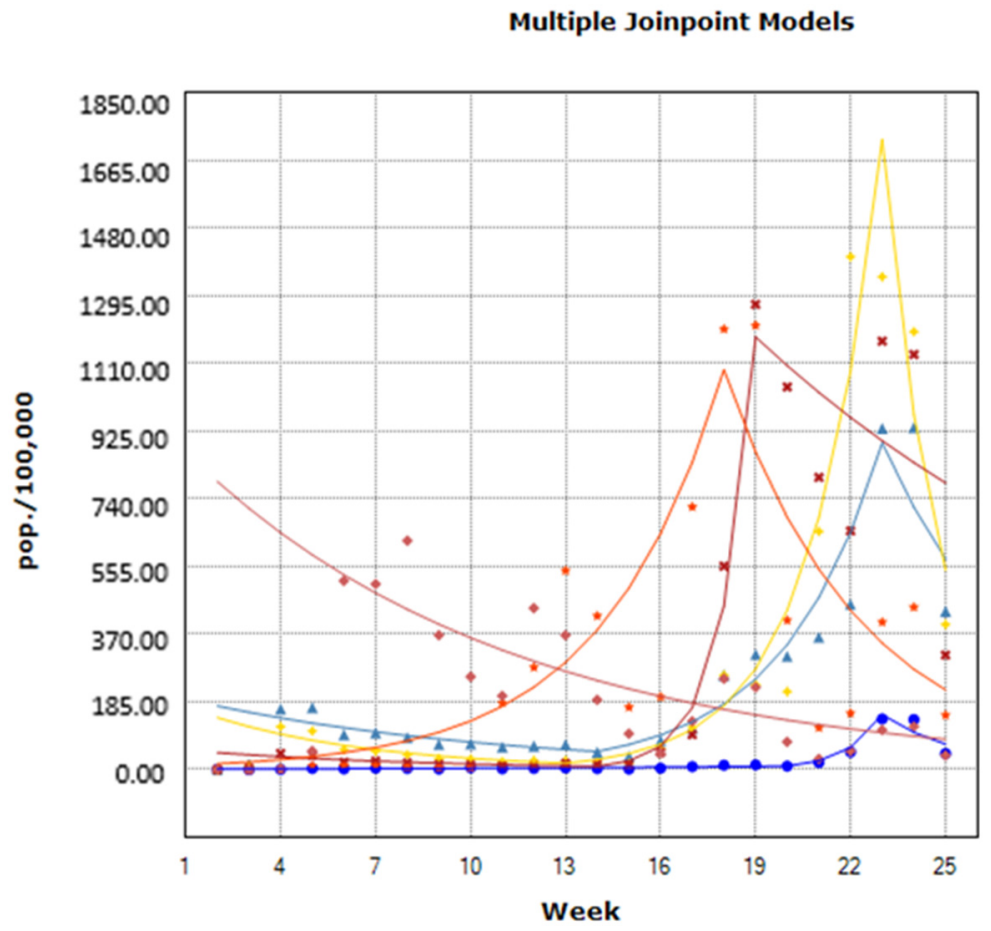

Figure 1. The weekly course of vaccinations per 100,000 population in Greece per age group (18-24 years: blue dots, 25-49 years: blue triangles, 50-59 years: yellow triangles, 60-69 years: red $x, 70-79$ years: orange asterisk, $>80$ years: red rhombus). The number on the y axis correspond to the number of vaccinations per 100,000 population. The $X$ axis corresponds to weeks of vaccination.

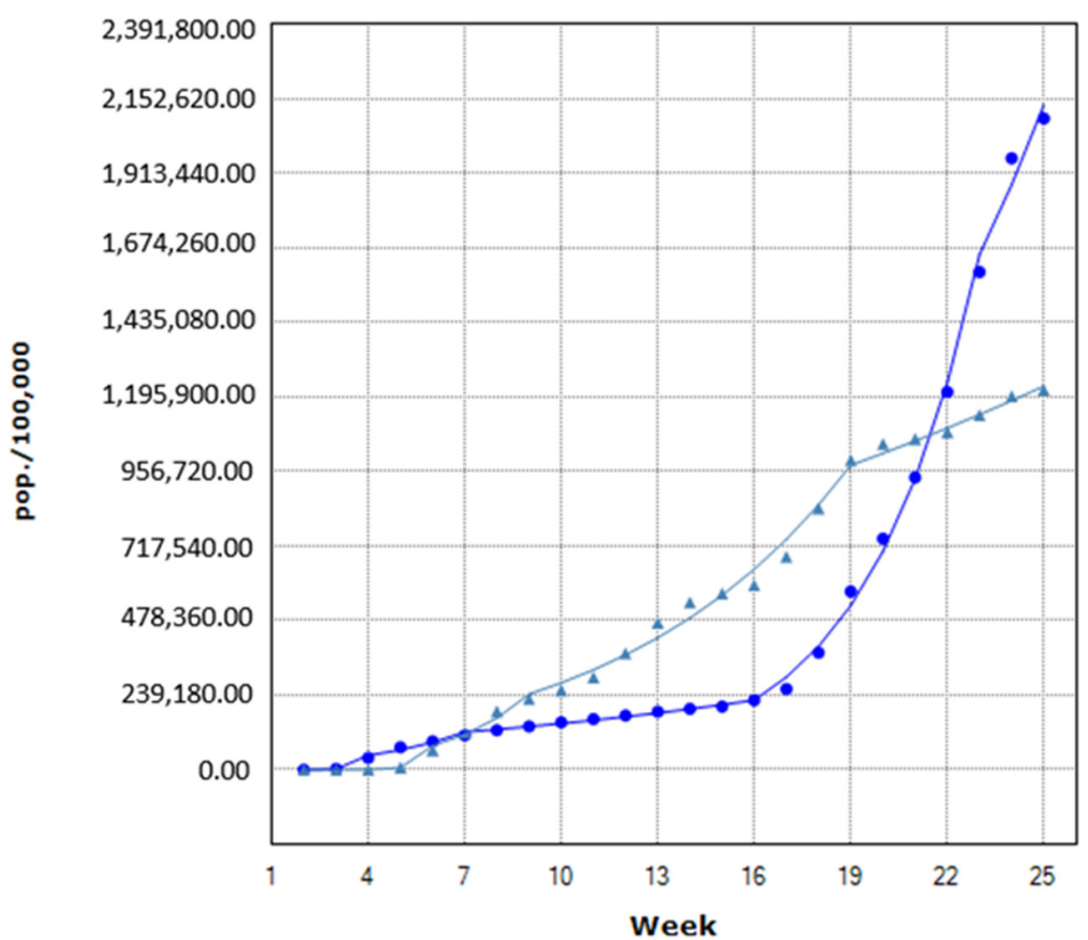

Figure 2. Average weekly vaccination trends by age in subjects $<70$ years (blue dots) and those $>70$ years (blue triangles).

As vaccination coverage accumulated nationwide, we observed that the weekly mean value of SARS-CoV-2 cases, COVID-19 ICU patients and COVID-19 deaths markedly declined (Figure 3). Figure 4 presents the results of joinpoint analysis for the trends in the 
rate of vaccinations, SARS-CoV-2 cases and COVID-19-related ICU admissions and deaths. In more detail, for all ages, the vaccination rate significantly increased from week 2 to week 6 by $85.70 \%$ (Cis: 28.1-169.1), and from week 7 to week 25 by 15.65\% (Cis: 14.9-16.4). The rate of SARS-CoV-2 cases increased significantly from week 2 to week 13 by $16.15 \%$ (Cis: 11.1-21.5), while from week 14 to week 25 the rate decreased significantly by $13.50 \%$ (Cis: -17.7--9.1). For the whole study duration, the rate of SARS-CoV-2 cases remained stable with a marginal difference of $0.9 \%$ (Cis: $-2.2-4.4$ ). For ICU admissions related to COVID-19, the rate decreased significantly by 7.41\% (Cis: $-13.5--0.9$ ) from week 2 to week 4 , followed by a significant increase of $17.22 \%$ (Cis: 15.1-19.4) from week 5 to week 11. The rate of ICU admissions remained stable during week 12 to week 16 (Cis: -0.7-4.6). The ICU admissions rate decreased significantly from week 17 to week 20 by $11.99 \%$ (Cis: $-15.9--7.9$ ) and from week 21 to week 25 by 16.77\% (Cis: $-20.2--13.2)$. For the whole study period, the rate of ICU admissions displayed a small reduction by $1.2 \%(-2.6-0.2)$, which did not reach statistical significance. Finally, the rate of COVID-19-related deaths increased significantly from week 2 to week 15 by $12.08 \%$ (Cis: $8.5-15.8$ ) and decreased significantly by $16.58 \%$ (Cis: $-22.4--10.4$ ) from week 16 to week 25 .

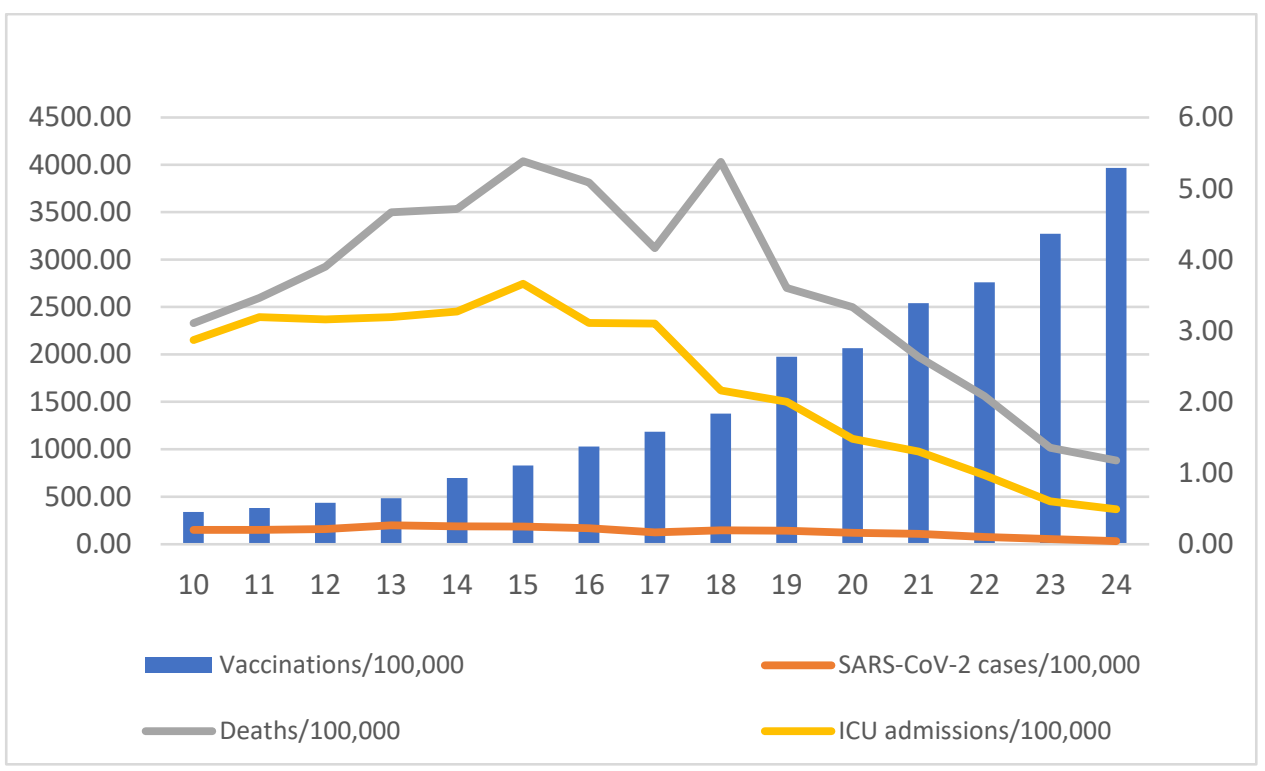

Figure 3. The course of vaccinations (per 100,000 population) in relation to weekly average of SARS-CoV-2 cases, COVID-19-related ICU admissions and COVID-19 deaths.

Figure 5 presents the average of vaccinations, weekly SARS-CoV-2 cases, ICU admissions and deaths for subjects $<70$ years. In this age group, the rate of SARS-CoV-2 cases increased statistically significantly by $16.18 \%$ from week 2 to week 13 (Cis: $10.8-21.8$ ) followed by a statistically significant decrease of $12.59 \%$ (Cis: $-16.9--8.1$ ) from week 14 to week 25 . ICU admissions decreased statistically significantly by $10.53 \%$ (Cis: $-18.4--1.9$ ) from week 2 to week 4, which was followed by a statistically significant increase of $20.39 \%$ (Cis:16.2-24.7) from week 5 to week 10 and a statistically significant increase of $6.1 \%$ (Cis: 0.5-12.1) from week 11 to week 14. For weeks 15 to 18, we did not observe a significant change in SARS-CoV-2 ICU admissions but there was a decrease of $16.09 \%$ between week 19 and the end of the study period. For COVID-19 deaths, we observed a statistically significant increase by 10.64\% (Cis: 7.2-14.2) from week 2 to week 17, which was followed by a statistically significant decrease by $22.03 \%$ (Cis: $-31--11.9$ ) from week 18 to week 25 . 

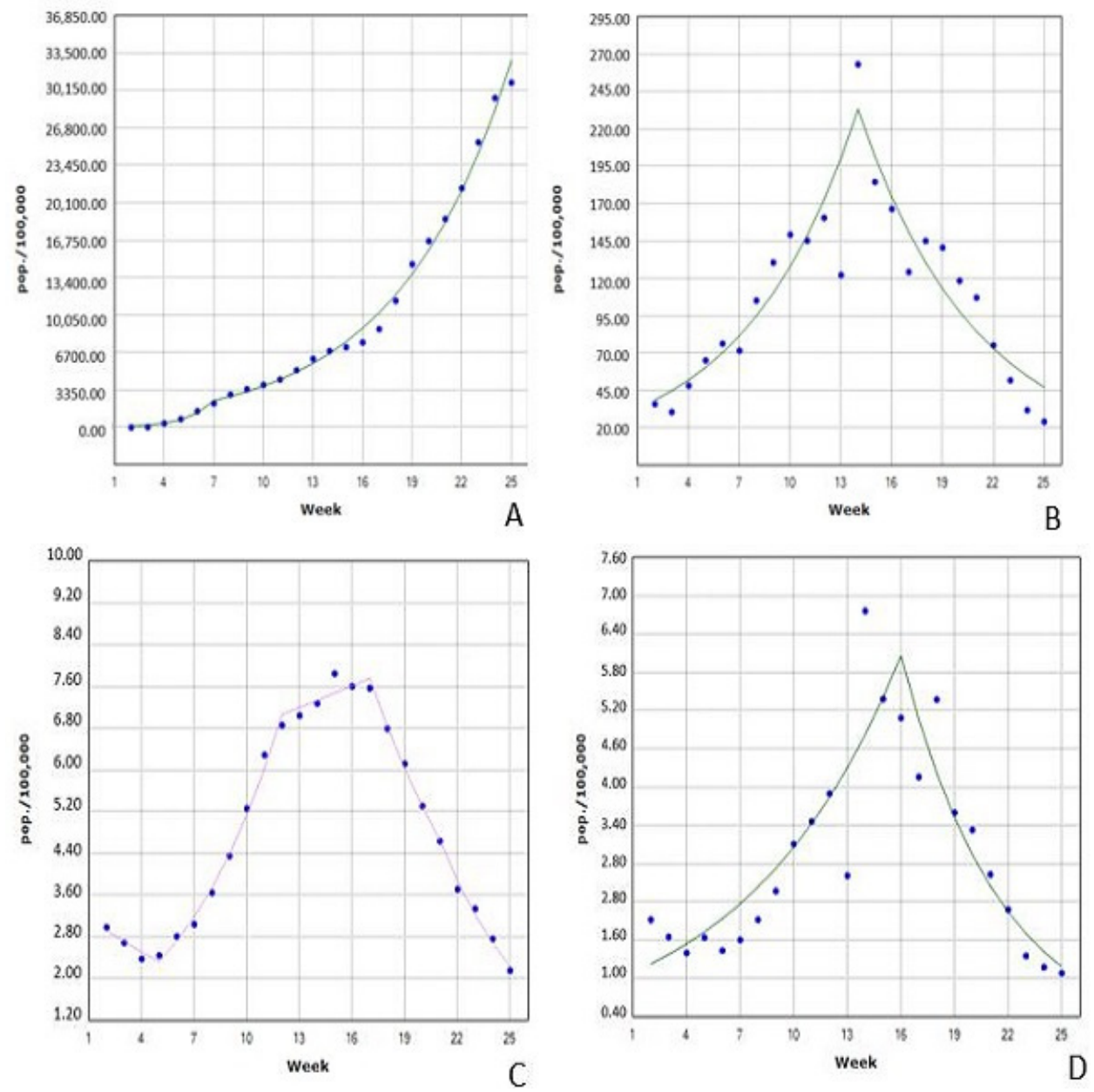

Figure 4. The course of vaccinations (per 100,000 population) (A), trends in the rate of SARS-CoV2 cases (B), COVID-19-related ICU admissions (C) and COVID-19-related deaths (D) during the study period.

Figure 6 displays the average of vaccinations, weekly COVID-19 cases, ICU admissions and deaths for subjects $\geq 70$ years. For SARS-CoV-2 cases, we observed an increase by 16.23\% (Cis: 12.1-20.5, $p<0.05$ ) from week 2 to week 13 while cases decreased significantly by $19.41 \%$ (Cis: $-23.4--15.2$ ) from week 14 to week 25 . For ICU admissions, we did not observe significant changes from week 2 to week 4, but cases increased significantly by 16.29\% (Cis: 13.3-19.4) form week 5 to week 11 and decreased by $14.3 \%$ (Cis: $-15.8--12.7$, $p<0.05$ ) from week 19 to week 25. From week 12 to week 14 and from week 15 to 18 they remained rather stable. For COVID-19 deaths, we observed a statistical increase by $11.9 \%$ (Cis: 8.3-15.6) from week 2 to week 15, followed by a statistically significant decrease by 17.1\% (Cis: -22.9--10.8) from week 16 to week 25. Overall (for the whole study period), in subjects $\geq 70$ we observed a decrease in new SARS-CoV-2 cases by $2.4 \%$ (Cis: $-5.2--0.4$ ), a decrease in ICU admissions by 1.2 (Cis: -2.8--0.4) and a decrease in COVID-19 deaths by 0.5 (Cis: $-3.7--2.8$ ). 


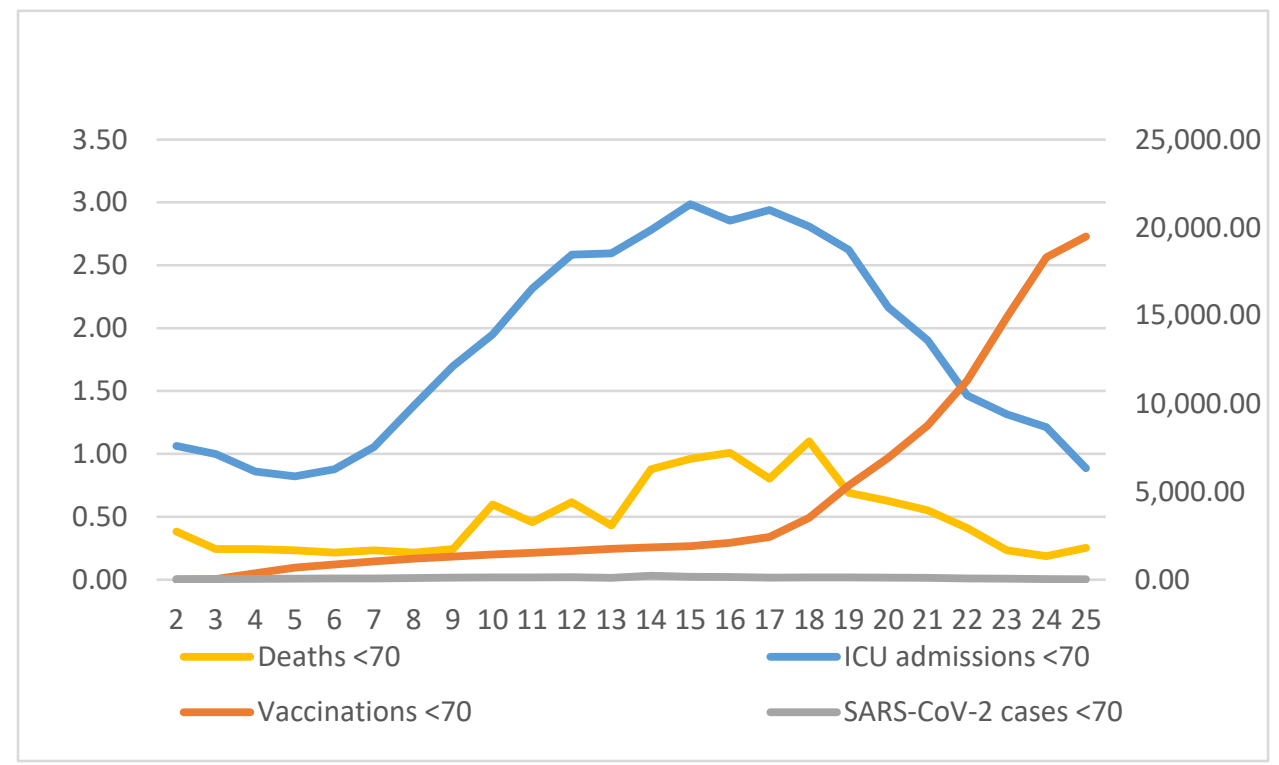

Figure 5. Average of vaccinations, weekly SARS-CoV-2 cases, ICU admissions and deaths for subjects $<70$ years. Data are expressed per 100,000 population. The left y axis corresponds to ICU admissions and deaths. The right $y$ axis refers to vaccinations and SARS-CoV-2 cases.

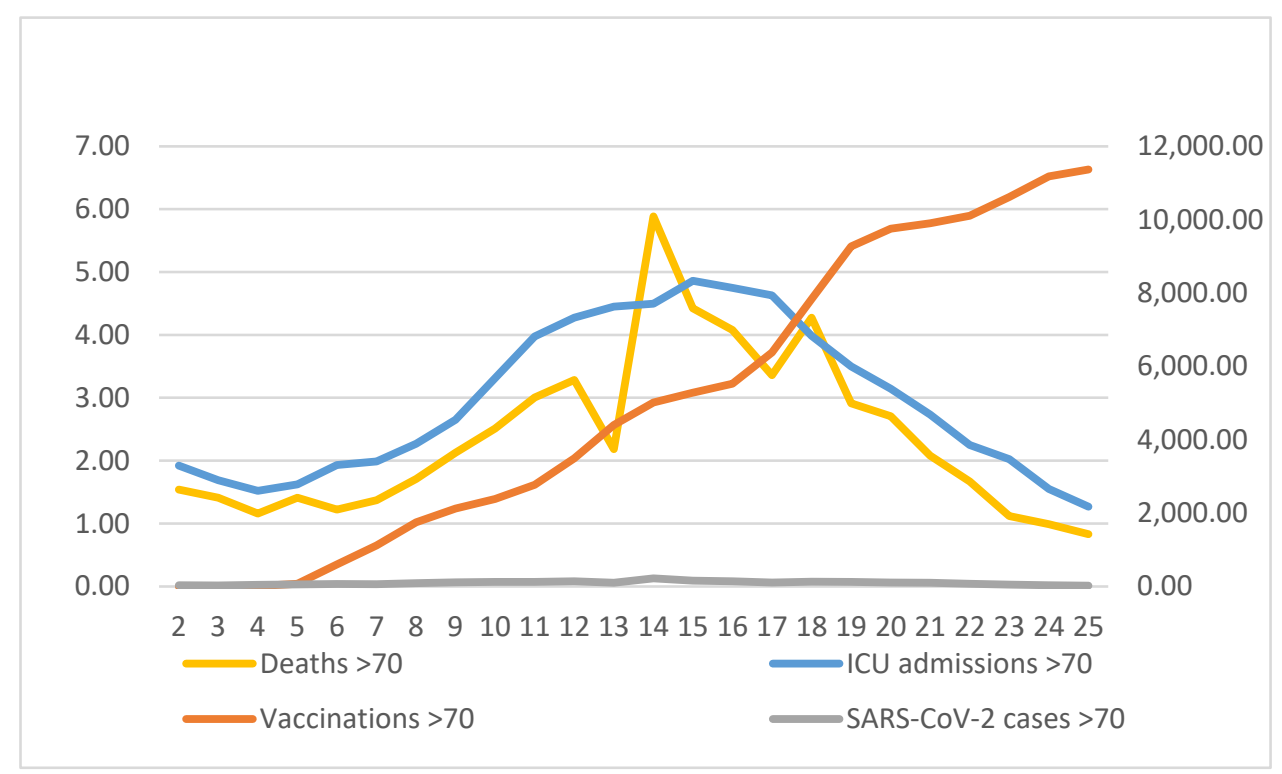

Figure 6. Average of vaccinations, weekly SARS-CoV-2 cases, ICU admissions and deaths for subjects $>70$ years. Data are expressed per 100,000 population. The left y axis corresponds to ICU admissions and deaths. The right y axis refers to vaccinations and SARS-CoV-2 cases.

Additionally, we analyzed COVID-19-related ICU admissions and death trends according to age and gender status. For COVID-19-related ICU admissions in the female population, for subjects 18-39 years old, we observed an increase by $143.9 \%$ (Cis: 25.5-374.0) from week 2 to week 4 and a further significant increase by 25.9\% (Cis: 6.5-47.7) from week 8 to week 13. For weeks 14-25, we observed a significant decrease by $12.8 \%$ (Cis: $-17.7--7.7)$. The rate remained stable for weeks 5-7. For females aged 40-64 years old, we observed a significant increase in COVID-19-related ICU admissions by $8.0 \%$ (Cis: $4.2-11.9$ ) for weeks 2-16, which was followed by a significant decrease by $13.7 \%$ (Cis: $-21.4--5.2$ ) for weeks 17-25. For females older than 65 years old, we observed a significant increase in ICU admissions by $10.8 \%$ (Cis: $8.1-13.5$ ) for weeks $2-15$ and a significant decrease by 
13.6\% (Cis: -17.7-9.3) for weeks 16-25. For COVID-19-related ICU admissions in the male population aged 18-39 years, we observed a significant decrease by $15.7 \%$ (Cis: - 26.5-3.2) for weeks 17-25. For this age group, for weeks $2-16$, we recorded a not significant increase by $4.1 \%$ in ICU admissions. For males aged 40-64 years, we observed a not significant increase by $7.2 \%$ for weeks $2-16$, which was followed by a significant decrease by $13.8 \%$ (Cis: -19--8.4) for weeks 17-25. Finally, for males older than 65 years, we observed a significant increase of ICU admissions by $20.2 \%$ (Cis: $8.1-33.6$ ) for weeks 7-10, which was followed by stability in the rate of admissions until week 16 . For weeks 17-25, we recorded a significant decrease by $13.5 \%$ (Cis: $-15.8--11.2$ ).

For COVID-19-related deaths, in the female population aged 18-39 years, we did not observe significant differences during the study period. For female subjects 40-64 years, deaths were rather stable for weeks $2-5$ while deaths increased by $21.0 \%$ (Cis: $8.9-34.5$ ) for week 6 to week 15 . For week 16 to week 25, we observed a significant decrease by $15.5 \%$ (Cis: $-23.2--7.1)$. For females older than 65 years, we observed stability of the death rate for week 2 to week 5. For week 6 to week 14 we observed a significant increase by $17.2 \%$ (Cis: 10.3-4.6), which was followed by a significant decrease of $14.6 \%$ (Cis: $-18.6--10.3$ ) for weeks 15-25. In the male population, deaths for ages 18-39 years were not recorded during the study period. For ages 40-64 years, we observed a significant increase of $10.0 \%$ (Cis: 6.4-13.7) for weeks 2-16, which was followed by a decrease of $17.3 \%$ (Cis: $-24.8--9.0$ ) for weeks 17-25. For males older than 65 years, we did not observe significant differences from week 2 to week 16, while for week 17 to week 25 we recorded a significant decrease in the death rate of $19.4 \%$ (Cis: $-27.1--10.9$ ).

\section{Discussion}

The data from this nationwide observational study underline the beneficial impact of the national vaccination campaign in Greece. As the vaccinations accumulated, we observed a significant decrease in SARS-CoV-2 cases, ICU admissions and deaths. The decreases were evident sooner in subjects $\geq 70$ years, who were vaccinated earlier than younger subjects. The declines in COVID-19 cases and outcomes occurred despite the fact that Greece was in a phase of reopening (following a national lockdown) since the 2nd week of the vaccination campaign.

The COVID-19 vaccines have shown effectiveness against the disease in randomized clinical trials and are now widely used in national vaccination campaigns worldwide. The BNT162b2 vaccine (Pfizer BioNTech) has 95\% efficacy against COVID-19 [5]. Vaccine effectiveness against symptomatic COVID-19 was estimated at 70.4\% for the ChAdOx1 nCoV-19 vaccine (AZD1222, Astra Zeneca) [7] and 94.1\% for the mRNA-1273 SARS-CoV2 vaccine (Moderna) [6]. Although randomized clinical trials provide important information regarding vaccine effectiveness, their population may have differences from the general population and thus it is essential to examine and report real-world effectiveness. Fukutami et al. [16] have used a public access COVID-19 database alongside a cases, vaccinations and COVID-19 (CaVaCo) tool to assess the efficiency of SARS-CoV-2 vaccination worldwide. The authors reported heterogeneity in the effects of vaccination across countries, with the majority of them exhibiting a positive correlation between COVID-19 vaccination, new SARS-CoV-2 cases and COVID-19 deaths. In Greece, we observed that as cumulative vaccination coverage increased, the weekly average incidence of SARS-CoV-2 cases and COVID-19 deaths decreased, after the 16th week of vaccination, despite the fact that the country was undertaking a phased reopening (that started at the 2 nd week of the vaccination campaign) following the nationwide lockdown. Our national population-level study adds important data on the effect of the vaccination campaign among the Greek population and provides insights into the real-life effects of vaccines in reducing the rates of SARS-CoV-2 cases and severe COVID-19 outcomes (ICU admissions and deaths).

We observed marked declines in SARS-CoV-2 incidence (as suggested by new SARSCoV-2 cases) and COVID-19 outcomes (i.e., ICU admissions and deaths) corresponding to increased vaccine uptake by the general population. Similar observations have been 
reported by Haas et al. in Israel [17], where COVID-19 vaccination proved effective in reducing symptomatic and asymptomatic SARS-CoV-2 infections, COVID-19-related severe and non-severe hospitalizations and COVID-19-related deaths. In a large community surveillance study in England, COVID-19 vaccination resulted in reductions in SARS-CoV-2 infections of $79 \%$ after the ChAdOx1 vaccine and of $80 \%$ after the BNT162b2 vaccine [18]. Similarly, in a retrospective study performed in an Italian province, the effectiveness of COVID-19 vaccination was estimated at $95 \%$ for the prevention of SARS-CoV-2 infections or COVID-19-related deaths [19]. In the same context, the real-world effectiveness of the vaccines against symptomatic disease or severe COVID-19 has been reported in elderly subjects [20]. Our results are in accordance with the aforementioned findings and provide further support for the impact of COVID-19 vaccination. In our study, the reductions in SARS-CoV-2 cases, ICU admissions and deaths occurred despite the fact that the country was in a phased reopening following the implementation of a nationwide lockdown of approximately 2 months' duration. Importantly, the COVID-19-related outcomes remained low even after the reopening had occurred, suggesting a positive impact of the COVID-19 vaccination campaign on public health.

Herd immunity occurs when a large percentage of the population is immune, resulting in decreased spread of the disease from person to person and thus protection of the whole community rather than immune subjects only. Historically, herd immunity was thought to be reached when approximately $65-70 \%$ of the population has been immunized [21] We observed a declining trend in new SARS-CoV-2 cases starting in the 14th week of the vaccination campaign when approximately $6.1 \%$ of the population was vaccinated. One study has reported that for some countries the infection rate after the vaccination campaign has an inverted U-shaped trend that is characterized by an increasing rate of infection after vaccination starts, which reaches a peak then declines as vaccinations accumulate [21]. Our results are in accordance with the aforementioned study and suggest that in some countries, presumably those that are underpopulated, like Greece, partial herd immunity may be reached earlier, and that the nationwide vaccination campaign should be intensive so as to quickly reach the turning point and prevent SARS-CoV-2 resurgence. The emergence of SARS-CoV-2 variants with increased transmissibility may lead to higher herd immunity thresholds, and efforts should be made to increase vaccine uptake in order to reduce SARS-CoV-2 transmission and morbidity [17].

Vaccine hesitancy results in a delay or refusal of vaccination despite vaccine availability and, in the COVID-19 era, it has emerged as a growing global threat to public health [22]. Although some populations, such as health care workers, have shown high acceptance of COVID-19 vaccination, other groups are more hesitant $[23,24]$. Safety concerns, doubts about the efficacy of the available vaccines and misinformation about the virus are some of the reasons underlying COVID-19 vaccine hesitancy, which may result in slower vaccination rates [25]. A study that was conducted in the USA presents statistically significant differences in vaccine hesitancy based on sociodemographic characteristics, with the highest prevalence of COVID-19 vaccine hesitancy found among African Americans, Hispanics, those who had children at home, individuals with lower education and incomes and rural dwellers [26].

Our study is not without limitations. We report reduced rates of SARS-CoV-2 cases, ICU admissions and deaths coinciding with the accumulation of vaccinations, but one cannot rule out the effect of potential cofounders and the positive contribution of other factors. One may consider the analysis of national surveillance data a limitation. The analysis of publicly available data is a common research method that may help answer research questions concerning global (or, in our case, national) responses to the novel coronavirus. The ecological design of our study cannot discriminate the impact of non-pharmaceutical interventions; however, it is notable that despite the reopening of the national lockdown we observed a significant drop in SARS-CoV-2 cases, ICU admissions and deaths as the vaccinations accumulated. Unfortunately, we do not have data on the vaccination status of, or the vaccines administered to, the patients with COVID-19, which could provide direct 
data regarding the effectiveness of the vaccines. Additionally, we do not have available demographic characteristics (age and gender) of the COVID-19 patients or the vaccinated subjects. Therefore, we cannot perform multivariate analysis to test for the possible effects of gender and age on COVID-19-related outcomes. We must also acknowledge that a significant limitation of the present study is the fact that the comparison of the trend of vaccinations with COVID-19 outcomes according to age relied on the grouping of age groups that were not identical, due to differences in reporting of data between the NPHO and ECDC that might have resulted in misclassification.

\section{Conclusions}

In conclusion, we observed a temporal association between vaccine uptake and reductions in the rate of SARS-CoV-2 cases, COVID-19-related ICU admissions and COVID-19related deaths. Our data suggest that high vaccine uptake may represent an efficient route towards normality, offering control of the SARS-CoV-2 pandemic as well as reducing the morbidity and mortality of COVID-19.

Author Contributions: Conceptualization, F.M. and K.I.G.; methodology, F.M. and I.C.L.; formal analysis, I.C.L.; data curation, I.C.L., D.P. and I.V.P.; writing - original draft preparation, F.M.; writingreview and editing, F.M., Z.D. and K.I.G. All authors have read and agreed to the published version of the manuscript.

Funding: This research received no external funding.

Institutional Review Board Statement: Ethical review and approval were waived for this study because it revised publicly available national surveillance data.

Informed Consent Statement: Patient consent was waived because we revised publicly available national surveillance data. No identifiable demographic or personal data were used in the present study.

Data Availability Statement: Data are available upon request.

Conflicts of Interest: The authors declare no conflict of interest.

\section{References}

1. World Meter. COVID-19 Coronavirus Pandemic. Available online: https://www.worldometers.info/coronavirus/ (accessed on 6 December 2021).

2. Johns Hopkins University. COVID-19 Dashboard by the Center for Systems Science and Engineering (CSSE). Available online: https:/ /www.arcgis.com/apps/dashboards/bda7594740fd40299423467b48e9ecf6 (accessed on 7 December 2021).

3. Peckham, H.; de Gruijter, N.M.; Raine, C.; Radziszewska, A.; Ciurtin, C.; Wedderburn, L.R.; Rosser, E.C.; Webb, K.; Deakin, C.T. Male sex identified by global COVID-19 meta-analysis as a risk factor for death and ITU admission. Nat Commun. 2020, 11, 6317. [CrossRef]

4. Vasileiou, E.; Simpson, C.R.; Shi, T.; Kerr, S.; Agrawal, U.; Akbari, A.; Bedston, S.; Beggs, J.; Bradley, D.; Chuter, A.; et al. Interim findings from first-dose mass COVID-19 vaccination roll-out and COVID-19 hospital admissions in Scotland: A national prospective cohort study. Lancet 2021, 397, 1646-1657. [CrossRef]

5. Polack, F.P.; Thomas, S.J.; Kitchin, N.; Absalon, J.; Gurtman, A.; Lockhart, S.; Perez, J.L.; Pérez Marc, G.; Moreira, E.D.; Zerbini, C.; et al. Safety and Efficacy of the BNT162b2 mRNA Covid-19 Vaccine. N. Engl. J. Med. 2020, 383, $2603-2615$. [CrossRef] [PubMed]

6. $\quad$ Baden, L.R.; El Sahly, H.M.; Essink, B.; Kotloff, K.; Frey, S.; Novak, R.; Diemert, D.; Spector, S.A.; Rouphael, N.; Creech, C.B.; et al. Efficacy and Safety of the mRNA-1273 SARS-CoV-2 Vaccine. N. Engl. J. Med. 2021, 384, 403-416. [CrossRef] [PubMed]

7. Voysey, M.; Clemens, S.A.C.; Madhi, S.A.; Weckx, L.Y.; Folegatti, P.M.; Aley, P.K.; Angus, B.; Baillie, V.L.; Barnabas, S.L.; Bhorat, Q.E.; et al. Safety and efficacy of the ChAdOx1 nCoV-19 vaccine (AZD1222) against SARS-CoV-2: An interim analysis of four randomised controlled trials in Brazil, South Africa, and the UK. Lancet 2021, 397, 99-111. [CrossRef]

8. Papagiannis, D.; Malli, F.; Raptis, G.D.; Papathanasiou, V.I.; Fradelos, C.E.; Daniil, Z.; Rachiotis, G.; Gourgoulianis, K.I. Assessment of Knowledge, Attitudes, and Practices towards New Coronavirus (SARS-CoV-2) of Health Care Professionals in Greece before the Outbreak Period. Int. J. Environ. Res. Public Health 2020, 17, 4925. [CrossRef] [PubMed]

9. Junjie, A.; Seng, J.J.B.; Seah, S.S.Y.; Low, L.L. COVID-19 Vaccine Hesitancy-A Scoping Review of Literature in High-Income Countries. Vaccines 2021, 9, 900. [CrossRef]

10. National Public Health Organization, (Greek CDC). Current State of COVID-19 Outbreak in Greece and Timeline of Key Containment Events. Available online: https:/ / eody.gov.gr/en/current-state-of-covid-19-outbreak-in-greece-and-timeline-ofkey-containment-events/ (accessed on 9 February 2022). 
11. Greek ECDC, Daily Surveillance Report of COVID-19. Available online: https://covid19-surveillance-report.ecdc.europa.eu/ archive-COVID19-reports/index.html (accessed on 9 February 2022).

12. Our World in Data. Available online: https:/ / ourworldindata.org/covid-cases.html (accessed on 9 February 2022).

13. European Centre for Disease Prevention and Control. Available online: https://www.ecdc.europa.eu/en (accessed on 10 October 2021).

14. Akhtar, S.; Al-Abkal, J.; Alroughani, R. Joinpoint Regression Analysis of Trends in Multiple Sclerosis Incidence in Kuwait: 1980-2019. Neuroepidemiology 2020, 54, 472-481. [CrossRef] [PubMed]

15. National Public Health Organization. Available online: https:/ / eody.gov.gr/en/npho/ (accessed on 10 October 2021).

16. Fukutani, K.F.; Barreto, M.L.; Andrade, B.B.; Queiroz, A.T.L. Correlation between SARS-CoV-2 Vaccination, COVID-19 Incidence and Mortality: Tracking the Effect of Vaccination on Population Protection in Real Time. Front. Genet. 2021, $12,679485$. [CrossRef] [PubMed]

17. Haas, E.J.; Angulo, F.J.; McLaughlin, J.M.; Anis, E.; Singer, S.R.; Khan, F.; Brooks, N.; Smaja, M.; Mircus, G.; Pan, K.; et al. Impact and effectiveness of mRNA BNT162b2 vaccine against SARS-CoV-2 infections and COVID-19 cases, hospitalisations, and deaths following a nationwide vaccination campaign in Israel: An observational study using national surveillance data. Lancet 2021, 397, 1819-1829. [CrossRef]

18. Pritchard, E.; Matthews, P.C.; Stoesser, N.; Eyre, D.W.; Gethings, O.; Vihta, K.D.; Jones, J.; House, T.; VanSteenHouse, H.; Bell, I.; et al. Impact of vaccination on new SARS-CoV-2 infections in the United Kingdom. Nat. Med. 2021, 27, 1370-1378 [CrossRef] [PubMed]

19. Flacco, M.E.; Soldato, G.; Martellucci, C.A.; Carota, R.; Di Luzio, R.; Caponetti, A. Interim Estimates of COVID-19 Vaccine Effectiveness in a Mass Vaccination Setting: Data from an Italian Province. Vaccines 2021, 9, 628. [CrossRef] [PubMed]

20. Bernal, J.L.; Andrews, N.; Gower, C.; Robertson, C.; Stowe, J.; Tessier, E.; Simmons, R.; Cottrell, S.; Roberts, R.; O’Doherty, M.; et al. Effectiveness of the Pfizer-BioNTech and Oxford-AstraZeneca vaccines on covid-19 related symptoms, hospital admissions, and mortality in older adults in England: Test negative case-control study. BMJ 2021, 373, n1088. [CrossRef] [PubMed]

21. Chen, Y.-T. The Effect of Vaccination Rates on the Infection of COVID-19 under the Vaccination Rate below the Herd Immunity Threshold. Int. J. Environ. Res. Public Health 2021, 18, 7491. [CrossRef] [PubMed]

22. Azarpanah, H.; Farhadloo, M.; Vahidov, R.; Pilote, L. Vaccine hesitancy: Evidence from an adverse events following immunization database, and the role of cognitive biases. BMC Public Health 2021, 21, 1686. [CrossRef] [PubMed]

23. Papagiannis, D.; Rachiotis, G.; Malli, F.; Papathanasiou, I.V.; Kotsiou, O.; Fradelos, E.C.; Giannakopoulos, K.; Gourgoulianis, K.I. Acceptability of COVID-19 Vaccination among Greek Health Professionals. Vaccines 2021, 9, 200. [CrossRef] [PubMed]

24. Marinos, G.; Lamprinos, D.; Georgakopoulos, P.; Patoulis, G.; Vogiatzi, G.; Damaskos, C.; Papaioannou, A.; Sofroni, A.; Pouletidis, T.; Papagiannis, D.; et al. Reported COVID-19 Vaccination Coverage and Associated Factors among Members of Athens Medical Association: Results from a Cross-Sectional Study. Vaccines 2021, 9, 1134. [CrossRef]

25. Dhama, K.; Sharun, K.; Tiwari, R.; Dhawan, M.; Emran, T.B.; Rabaan, A.A.; Alhumaid, S. COVID-19 vaccine hesitancy-Reasons and solutions to achieve a successful global vaccination campaign to tackle the ongoing pandemic. Hum. Vaccines Immunother. 2021, 17, 3495-3499. [CrossRef]

26. Khubchandani, J.; Sharma, S.; Price, J.H.; Wiblishauser, M.J.; Sharma, M.; Webb, F.J. COVID-19 Vaccination Hesitancy in the United States: A Rapid National Assessment. J. Community Health 2021, 46, 270-277. [CrossRef] [PubMed] 